\title{
AN INTEGRAL REPRESENTATION OF A NORMAL FUNCTIONAL ON A VON NEUMANN ALGEBRA $\left({ }^{1}\right)$
}

\author{
BY \\ HERBERT HALPERN
}

1. Introduction. Let $\mathscr{A}$ be a von Neumann algebra with center $\mathscr{Z}$ on a Hilbert space $H$; a positive function $g$ on $\mathscr{A}$ is included in a positive functional $f$ on $\mathscr{A}$ (notation: $g<f$ ) if there is a scalar $\alpha>0$ such that $f-\alpha g$ is a positive functional on $\mathscr{A}$. A nonzero positive functional on $\mathscr{A}$ which includes only scalar multiples of itself is said to be irreducible. In this paper we shall call a positive functional $f$ on $\mathscr{A}$ generalized irreducible if whenever $g$ is a positive functional such that $g<f$ there is an element $A_{0}$ in $\mathscr{A}^{+}$, the cone of positive elements of $\mathscr{A}$, such that $f\left(A_{0} A\right)=$ $g(A)$, for all $A$ in $\mathscr{A}$. A positive functional $f$ on $\mathscr{A}$ is said to be normal if for each monotonely increasing net $\left\{A_{d} \mid d \in D\right\}$ with least upper bound $A$, we have $f(A)=$ $\operatorname{lub}\left\{f\left(A_{d}\right) \mid d \in D\right\}$. In the present paper we obtain a representation theorem for a normal generalized irreducible functional $f$ on a von Neumann algebra $\mathscr{A}$ of Type I. The representation has the form (A) $f(A)=\int_{Z} f_{\zeta}(A) d v(\zeta)$ where

(1) $Z$ is the spectrum of the center $\mathscr{Z}$;

(2) $\nu=v_{h}(h \in H)$ is the so-called spectral measure on $Z$ given by $f(A)=w_{h}(A)=$ $\int_{Z} \hat{A}(\zeta) d \nu(\zeta)$ where $A \in \mathscr{Z}$ and the function $\hat{A}$ is the image of $A$ in the algebra of all continuous complex-valued functions on $Z$ under the Gelfand representation; and

(3) the $f_{\zeta}$ for $\zeta$ in the support $Y$ of $\nu$ are positive functionals such that for each fixed $A$ in $\mathscr{A}$ the map $\zeta \rightarrow f_{\zeta}(A)$ is continuous. We shall show that the functionals $f_{\zeta}$ have special properties. We also prove that normal functional $f$ obtained from a representation of the form (A) in which the functionals $f_{\zeta}$ have this special property is generalized irreducible.

These theorems may be viewed as a generalization of certain results of Tomita [6]. Tomita studied positive functionals $f$ on a $C^{*}$-algebra $\mathscr{A}$ with identity and with center $\mathscr{Z}$ with the property: if $g<f$, there is an $A_{0} \in \mathscr{Z}^{+}$such that $g(A)=$ $f\left(A A_{0}\right)$ for all $A \in \mathscr{A}$. These functionals are called centrally irreducible functionals. Some of the lemmas necessary for this generalization give results on centrally reducible functionals with little additional effort. We therefore briefly indicate in what direction this effort should be applied.

Let $\mathscr{A}$ be a von Neumann algebra with center $\mathscr{Z}$ on a Hilbert space $H$; let $Z$

Received by the editors October 25, 1965.

( ${ }^{1}$ This article consists of part of the author's doctoral dissertation submitted to the Department of Mathematics of Harvard University. 
be the spectrum of $\mathscr{Z}$. For each $\zeta \in Z$ the two-sided closed ideal in $\mathscr{A}$ generated by

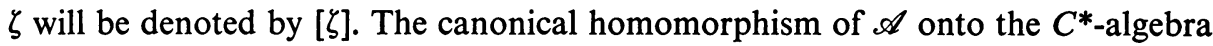
$\mathscr{A} /[\zeta]$ will be written as $\Psi_{\zeta}$ while $\Psi_{\zeta}(A)$ will be written as $A(\zeta)$ for each $A \in \mathscr{A}$. Glimm has proved that for each fixed $A$ in $\mathscr{A}$, the function $\zeta \rightarrow\|A(\zeta)\|$ is continuous on $Z$.

2. Centrally irreducible functionals. A projection $E$ in $\mathscr{A}$ is abelian if the von Neumann algebra $E \mathscr{A} E$ is commutative. If $P$ is the central support of $E$ (i.e., the smallest projection in $\mathscr{Z}$ such that $P \geqq E$ ), the map $\Phi(A)=A E$ of $\mathscr{Z} P$ onto $E \mathscr{A} E$ is an isomorphism. We define the linear function $\tau_{E}(A)$ on $\mathscr{A}$ as $\tau_{E}(A)=\Phi^{-1}(E A E)$.

Proposition 2.1. For each $\zeta$ in the set $\{\zeta \in Z \mid \hat{P}(\zeta)=1\}$, the functional $f(A)=$ $\tau_{E}(A)^{\wedge}(\zeta)$ on $\mathscr{A}$ is irreducible.

Proof. The verification that $f$ is a nonzero positive functional is straightforward. Let $g$ be a positive functional on $\mathscr{A}$ included in $f$. Since $f([\zeta])=0$, $g([\zeta])=0$. There is a functional $g_{1}$ on $\mathscr{A}(\zeta)$ such that $g_{1}(A(\zeta))=g(A)$, for all $A$ in $\mathscr{A}$. Furthermore, $g(I-E)=0$; this means that $g(A)=g(E A E)$, for all $A$ in $\mathscr{A}$. Therefore, we have

$$
\begin{aligned}
g(A)=g(E A E) & =g\left(\tau_{E}(A) E\right) \\
& =g_{1}\left(\tau_{E}(A)^{\wedge}(\zeta) E(\zeta)\right) \\
& =f(A) g_{1}(E(\zeta))=f(A) g(E),
\end{aligned}
$$

for each $A$ in $\mathscr{A}$. This shows that $g$ is a scalar multiple of $f$ and hence that $f$ is irreducible on $\mathscr{A}$. Q.E.D.

If $f$ is a positive functional on a $C^{*}$-algebra with identity, let $L(f)$ be the closed left-ideal $L(f)=\left\{A \in \mathscr{A} \mid f\left(A^{*} A\right)=0\right\}$. The Hilbert space which is the completion of the left $\mathscr{A}$-module $\mathscr{A}-L(f)$ under the inner product $(A-L(f), B-L(f))=f\left(B^{*} A\right)$ is denoted by $H(f)$. The operator $\Phi(A)(A \in \mathscr{A})$ on $H(f)$ which is the extension of $\Phi(A)(B-L(f))=A B-L(f)$ to $H(f)$ defines a continuous linear operator on $H(f)$. The homomorphism $A \rightarrow \Phi(A)$ of $\mathscr{A}$ into the *-algebra of bounded operators on $H(f)$ is called the canonical representation of $\mathscr{A}$ on $H(f)$ induced by $f$.

LEMMA 2.2. If $f$ is a centrally reducible functional on a $C^{*}$-algebra $\mathscr{A}$ with center $\mathscr{Z}$, then the commutator $\Phi(\mathscr{A})^{\prime}$ of $\Phi(\mathscr{A})$ on $H(f)$ is $\Phi(\mathscr{Z})$. Here $\Phi$ denotes the canonical representation on $H(f)$ induced by $f$.

Proof. We must prove that $\Phi(\mathscr{A})^{\prime} \subset \Phi(\mathscr{Z})$. Let $B \in\left(\Phi(\mathscr{A})^{\prime}\right)^{+}$; let $h$ be a cyclic vector in $H(f)$ under $\Phi(\mathscr{A})$ such that $(\Phi(A) h, h)=f(A)$, for all $A$ in $\mathscr{A}$. Then the functional $g(A)=(\Phi(A) \cdot B h, h)$ on $\mathscr{A}$ is majorized by $f$. Consequently, there is a 
$C \in \mathscr{Z}^{+}$such that $(\Phi(A) B h, h)=(\Phi(A) \Phi(C) h, h)$, for all $A$ in $\mathscr{A}$. Thus $\Phi(C)=B$ and $\left(\Phi(\mathscr{A})^{\prime}\right)+\subset \Phi(\mathscr{Z})$. So $\Phi(\mathscr{A})^{\prime} \subset \Phi(\mathscr{Z})$.

THEOREM 2.3. Let $\mathscr{A}$ be a von Neumann algebra with center $\mathscr{Z}$ on a Hilbert space $H$. A normal functional $f$ on $\mathscr{A}$ is centrally irreducible if and only if $f=w_{h}$ where $h$ is a vector in $H$ such that the cyclic projection $E\left(A^{\prime}, h\right)$ corresponding to the closed subspace closure $\left\{A^{\prime} h \mid A^{\prime} \in \mathscr{A}^{\prime}\right\}$ is abelian.

Proof. Let $f$ be a centrally irreducible functional on $\mathscr{A}$ and let $E$ be the support of $f$. Let $\Phi$ be the canonical representation of $f$ on $H(f)$ induced by $f$ and let $\mathscr{A} P$ be the kernel of $\Phi$ where $P$ is a central projection. Then there is a $k \in H(f)$ such that $f(A)=(\Phi(A) k, k)$, for all $A \in \mathscr{A}$. We have that $\Phi(E)$ is the support of $w_{k}$ on $\Phi(\mathscr{A})$. Since $\Phi(E) \Phi(\mathscr{A})^{\prime} \Phi(E)=\Phi(E) \Phi(\mathscr{Z}) \Phi(E)$, we have that $\Phi(E) \Phi(\mathscr{A}) \Phi(E)=$ $\Phi(E) \Phi(\mathscr{Z}) \Phi(E)$. Thus, $\Phi(E)$ is an abelian projection in $\Phi(\mathscr{A})$. This means that $E$ is an abelian projection in $\mathscr{A}$ because $E \leqq I-P$. We have that $f \mid E \mathscr{A} E$ is given by $f\left|E \mathscr{A} E=w_{h}\right| E \mathscr{A} E$ for some $h \in E(H)$. Thus, $f=w_{h}$ and $E=E\left(A^{\prime}, h\right)$ is an abelian projection.

Conversely let $f=w_{h}$ where $E=E\left(\mathscr{A}^{\prime}, h\right)$ is abelian. Let $g$ be a positive functional on $\mathscr{A}$ majorized by $f$. For each $A$ in $\mathscr{A}$ we have $g(E A E)=g(A)$. The functional $g \mid E \mathscr{A} E$ is majorized by $f \mid E \mathscr{A} E$. There is a $B$ in $(E \mathscr{A} E)^{+}=(\mathscr{Z} E)^{+}$such that $g(E A E)=f(B \cdot E A E)$ for all $A$ in $\mathscr{A}$. If $B=C E$ where $C$ is a member of $\mathscr{Z}^{+}$, then $g(A)=f(C A)$ for all $A$ in $\mathscr{A}$. Q.E.D.

Let $f$ be a centrally irreducible functional on a $C^{*}$-algebra $\mathscr{A}$ with identity $I$ and center $\mathscr{Z}$; then the commutator $\Phi(\mathscr{A})^{\prime}$ of the image $\Phi(\mathscr{A})$ of $\mathscr{A}$ under the canonical representation $\Phi$ of $\mathscr{A}$ on $H(f)$ induced by $f$ is equal to $\Phi(\mathscr{Z})$. The functional $w_{h}(h=I-L(f))$ on the von Neumann algebra $\Phi(\mathscr{A})^{\prime \prime}$ on $H(f)$ generated by $\Phi(\mathscr{A})$ is representable as

$$
w_{h}(B)=\int_{z_{1}} \tau_{E}(B)^{\wedge}(\zeta) d \nu_{1}(\zeta) \quad\left(B \in \Phi(\mathscr{A})^{\prime \prime}\right) .
$$

The measure $\nu_{1}$ is the spectral measure $\nu_{h}$ on the spectrum $Z_{1}$ of $\Phi(\mathscr{Z})$, and the projection $E$ is the cyclic projection $E=E\left(\Phi(\mathscr{A})^{\prime}, h\right)$. The functionals $f_{\zeta}(B)=$ $\tau_{E}(B)^{\wedge}(\zeta)\left(\zeta \in Z_{1}\right)$ are irreducible on $\Phi(\mathscr{A})^{\prime \prime}$ and therefore the $f_{\zeta}\left(\zeta \in Z_{1}\right)$ are elements of the pure state space of $\Phi(\mathscr{A})$ [2]. The representation $\Phi$ of $\mathscr{Z}$ onto $\Phi(\mathscr{Z})$ induces a homeomorphism of $Z_{1}$ into $Z$; the representation for

$$
w_{h}(\Phi(A))=f(A) \quad(A \in \mathscr{A})
$$

can be carried over to an integral representation on the spectrum $Z$ of $\mathscr{Z}$ of the form $\int_{z} g_{\zeta}(A) d \nu(\zeta)$. Each of the functionals $g_{\zeta}(\zeta \in$ support $\nu)$ is in the pure state space of $\mathscr{A}$; and for each fixed $A$ in $\mathscr{A} \zeta \rightarrow g_{\zeta}(A)$ is continuous on the support of $\nu$. These steps will be discussed in more detail in the ensuing theorems. 
3. Generalized irreducible functionals. Let $\mathscr{A}$ be an algebra of continuous linear operators on a Hilbert space $H$. The commutator of $\mathscr{A}$ is the set $\mathscr{A}^{\prime}$ of all continuous linear operators $A^{\prime}$ on $H$ such that $A A^{\prime}=A^{\prime} A$ for all $A$ in $\mathscr{A}$. If $h$ is a vector in $H$, the cyclic projection $E(\mathscr{A}, h)$ generated by $\mathscr{A}$ and $h$ is that projection which corresponds to the subspace of $H$ given by closure $\{A h \mid A$ in $\mathscr{A}\}$. A nonzero vector $h$ in $H$ is called a trace element for the algebra $\mathscr{A}$ if $(A B h, h)=(B A h, h)$ for all $A, B$ in $\mathscr{A}$.

We first clarify the notion of a generalized irreducible functional in the next proposition.

Proposition 3.1. Let $\mathscr{A}$ be a von Neumann algebra on a Hilbert space H. The vector functional $w_{h}$ is generalized irreducible on $\mathscr{A}$ if and only if $h$ is a trace element for $E \mathscr{A} E$ where $E=E\left(\mathscr{A}^{\prime}, h\right)$.

Proof. The statement that $w_{h}$ is generalized irreducible if $h$ is a trace element on $E \mathscr{A} E$ is known [1, p. 96, Proposition 5]. We proceed with the proof of the converse. Let $w_{h}$ be generalized irreducible on $\mathscr{A}$ and let $E^{\prime}$ be the projection in $\mathscr{A}^{\prime}$ given by $E^{\prime}=E(\mathscr{A}, h)$. If $A^{\prime}$ is an element in $\left(\mathscr{A}^{\prime}\right)^{+}$, we have that $g(A)=w_{h}\left(A^{\prime} A\right)$ is a positive functional on $\mathscr{A}$ included in $w_{h}$. Thus, there is an element $A_{0}$ in $\mathscr{A}^{+}$ such that $g(A)=w_{h}\left(A A_{0}\right)$ for all $A$ in $\mathscr{A}$. This means

$$
\left(\left(A_{0}-A^{\prime}\right) h, A h\right)=0
$$

for all $A$ in $\mathscr{A}$. Since $\{A h \mid A \in \mathscr{A}\}$ is dense in $E^{\prime}(H)$, we have

$$
E^{\prime}\left(A_{0}-A^{\prime}\right) E^{\prime} h=0 .
$$

Therefore, for any $A^{\prime}$ in $\mathscr{A}^{\prime}$, there is an element $A_{0}$ in $\mathscr{A}$ such that

$$
E^{\prime} A^{\prime} E^{\prime} h=E^{\prime} A_{0} E^{\prime} h
$$

and

$$
E^{\prime} A^{*} E^{\prime} h=E^{\prime} A_{0}^{*} E^{\prime} h
$$

These relations show that $h$ is a trace element for $E^{\prime} \mathscr{A}^{\prime} E^{\prime}$. Indeed, for $A^{\prime}, B^{\prime}$, in $\mathscr{A}^{\prime}$ we have

$$
\left(B^{\prime} A^{\prime} h, h\right)=\left(B^{\prime} A_{0} h, h\right)=\left(B^{\prime} h, A_{0}^{*} h\right)=\left(B^{\prime} h, A^{\prime *} h\right)=\left(A^{\prime} B^{\prime} h, h\right)
$$

where $A_{0}$ is an element of $\mathscr{A}$ given by $E^{\prime} A_{0} E^{\prime} h=E^{\prime} A^{\prime} E^{\prime} h$ and $E^{\prime} A_{0}^{*} E^{\prime} h=$ $E^{\prime} A^{\prime *} E^{\prime} h$.

By the first part of the theorem $w_{h}$ is generalized irreducible on $\mathscr{A}^{\prime}$; a second application of the preceding argument shows $w_{h}$ is generalized irreducible on $\mathscr{A}$.

From the preceding proposition the next decomposition follows immediately. 
THEOREM 3.2. Let $\mathscr{A}$ be a von Neumann algebra of Type I with center $\mathscr{Z}$ on a Hilbert space $H$. If $f$ is a generalized irreducible normal functional on $\mathscr{A}$, we may write (A) $f(A)=\int f_{\zeta}(A) d \nu(\zeta)$, for all $A$ in $\mathscr{A}$. Here

(1) $\nu$ is the spectral measure $\nu_{h}$ on the spectrum $Z$ of $\mathscr{Z}$; the vector $h$ arises from restricting $f$ to $\mathscr{Z}$;

(2) $f_{\zeta}$ is a state for each $\zeta$ in the support $Y$ of $\nu$ and $\zeta \rightarrow f_{\zeta}(A)$ is continuous on $Y$ for each fixed $A$ in $\mathscr{A}$;

(3) for each $\zeta$ in $Y, f_{\zeta}(A)=\hat{A}(\zeta)$ for all $A$ in $\mathscr{Z}$;

(4) except on a nowhere dense set $N$ in $Y, f_{\zeta}$ is a finite sum of irreducible functionals on $\mathscr{A}$; and

(5) except on $N, f_{\zeta}$ is generalized irreducible on $\mathscr{A}$.

Proof. We prove the parts (1), (2), (3) without using the hypothesis that $\mathscr{A}$ is of Type I.

Let $\Phi$ be the canonical representation of $\mathscr{A}$ on $H(f)$ induced by $f$. The map $\Phi$ is normal and therefore $\Phi(\mathscr{A})$ is a von Neumann algebra on $H(f)$. Let $k$ be the cyclic vector in $H(f)$ under $\Phi(\mathscr{A})$ such that $(\Phi(A) k, k)=f(A)$ for all $A$ in $\mathscr{A}$. If $g$ is a positive functional included in $w_{k}$, it is easy to see that there is a $B \in \Phi(\mathscr{A})^{+}$ such that $g(A)=w_{k}(B A)$ for all $A$ in $\Phi(\mathscr{A})$. Thus, $w_{k}$, is generalized irreducible on the algebra $\Phi(\mathscr{A})$. Let $F$ be the projection in $\Phi(\mathscr{A})$ given by $F=E\left(\Phi(\mathscr{A})^{\prime}, k\right)$. There is a projection $Q$ in $\mathscr{Z}$ such that the kernel of $\Phi$ is $A(I-Q)$. The unique projection $E$ in $\mathscr{A} Q$ such that $\Phi(E)=F$ is the support of $f$.

We claim that $f$ is a faithful finite normal trace on $E \mathscr{A} E$. That $f$ is faithful and finite needs no verification. To prove $f$ is a trace on $E \mathscr{A} E$, let $A$ and $B$ be members of $E \mathscr{A} E$. We have

$$
\begin{aligned}
f(A B) & =(\Phi(A B) k, k) \\
& =(\Phi(A) \Phi(B) k, k) \\
& =(\Phi(B) \Phi(A) k, k) \\
& =(\Phi(B A) k, k)=f(B A) .
\end{aligned}
$$

Thus, $f$ is a trace on $E \mathscr{A} E$.

We have proved that $E \mathscr{A} E$ is a finite von Neumann algebra. If $\mathscr{A}$ is of Type I, so is $E \mathscr{A} E$. The algebra $E \mathscr{A} E$ possesses a canonical \#-map $A \rightarrow A^{\#}$ of $E \mathscr{A} E$ onto the center $\mathscr{Z}_{1}$ of $E \mathscr{A} E$ [1]. We may write for all $A$ in $E \mathscr{A} E$

$$
f(A)=f\left(A^{\#}\right)=\int A^{\# \wedge}\left(\zeta_{1}\right) d \nu_{1}\left(\zeta_{1}\right)
$$

where $\nu_{1}$ is the spectral measure on the spectrum $Z_{1}$ of $\mathscr{Z}_{1}$. The measure arises from restricting $f$ to $\mathscr{Z}_{1}$. 
Let $P$ be the central support of $E$. There is an isomorphism of $\mathscr{Z} P$ into $\mathscr{Z}_{1}$ given by $A \rightarrow A E$, for $A \in \mathscr{Z} P$. There is a homeomorphism $\rho$ of the spectrum $Z_{1}$ of $\mathscr{Z}_{1}$ onto the spectrum

$$
Y=\{\zeta \in Z \mid \hat{P}(\zeta)=1\} \text { of } \mathscr{Z P} \text { such that }
$$

the map $A \rightarrow A E$ of $\mathscr{Z} P$ to $\mathscr{Z}_{1}$ is given by $\hat{A} \rightarrow \hat{A} \cdot \rho$ when $\mathscr{Z} P$ and $\mathscr{Z}_{1}$ are identified by the Gelfand map with the algebras of all continuous complex-valued functions on $Y$ and on $Z_{1}$ respectively.

We use $\rho^{-1}=\eta$ to obtain a representation on $Y$. For each $\zeta \in Y$, we define $f_{\zeta}(A)=(E A E)^{\# \wedge}(\eta(\zeta))$, for each $A$ in $\mathscr{A}$. It is easy to see that $f_{\zeta}$ is a state for each $\zeta \in Y$, and that for each fixed $A$ in $\mathscr{A}$, the function $\zeta \rightarrow f_{\zeta}(A)$ is continuous on $Y$. Furthermore, if $A \in \mathscr{Z}$,

$$
\begin{aligned}
f_{\zeta}(A)=(E A E)^{\# \wedge}(\eta(\zeta)) & =(A E)^{\wedge}(\eta(\zeta)) \\
& =(A P)^{\wedge}(\rho \cdot \eta(\zeta)) \\
& =\hat{A}(\zeta),
\end{aligned}
$$

since $P^{\wedge}(\zeta)=1$ for $\zeta \in Y$. Therefore, $f_{\zeta}$ satisfies condition (3).

Let $\nu$ be the Radon measure on $Z$ with support $Y$ given by the formula

$$
\nu(X)=\nu_{1}(\eta(X)) \text { for each Borel set } X \subset Y .
$$

Defining $f_{\zeta}=0$ for $\zeta \notin Y$, we have

$$
f(A)=\int_{z} f_{\zeta}(A) d \nu(\zeta)
$$

for all $A$ in $\mathscr{A}$.

We now show that $\nu$ is a spectral measure. There is an element $h$ in $H$ such that $f\left|\mathscr{Z}=w_{h}\right| \mathscr{Z}$. We have

$$
f(A)=\int \hat{A}(\zeta) d \nu(\zeta)=w_{h}(A)
$$

for all $A$ in $\mathscr{A}$. By definition $\nu=\nu_{h}$.

We now employ the hypothesis that $\mathscr{A}$ is of Type I to obtain properties (4) and (5). First we construct the nowhere dense set $N$ in $Y$. Let

$$
E \mathscr{A} E=\prod\left\{(E \mathscr{A} E) \cdot P_{d} \mid d \in D\right\}
$$

where the $P_{d}$ are mutually orthogonal central projections in $E \mathscr{A} E$ with least upper bound $E$ such that $(E \mathscr{A} E) \cdot P_{d}$ is homogeneous for each $d \in D$. Let

$$
\begin{aligned}
S_{d} & =\left\{\zeta_{1} \in Z_{1} \mid \hat{P}_{d}\left(\zeta_{1}\right)=1\right\} \text { and let } \\
S & =\bigcup\left\{S_{d} \mid d \in D\right\} .
\end{aligned}
$$


The set $S$ is open and dense in $Z_{1}$; we set $N_{1}$ equal to the complement of $S$ in $Z_{1}$. The set $N_{1}$ is nowhere dense in $Z_{1}$ and thus $N=\rho\left(N_{1}\right)$ is nowhere dense in $Y$.

For each $\zeta \in Y-N$ we must prove that $f_{\zeta}$ satisfies properties (4) and (5). Let $P_{0}$ be the unique projection in $\left\{P_{d} \mid d \in D\right\}$ such that $\hat{P}_{0}\left(\zeta_{0}\right)=1$ where $\zeta_{0}=\eta(\zeta)$. Since the algebra $(E \mathscr{A} E) \cdot P_{0}$ is finite and homogeneous, there are equivalent orthogonal abelian projections $E_{1}, E_{2}, \ldots, E_{n}$ such that $E_{1}+E_{2}+\cdots+E_{n}=E$. Let $U_{j k}$ $(1 \leqq j, k \leqq n)$ be partial isometric operators in $(E \mathscr{A} E) \cdot P_{0}$ such that

(1) $U_{j k} U_{l m}=\delta_{m j} U_{l k}$, where $\delta$ is the Kronecker delta;

(2) $U_{j k}^{*}=U_{k j}$; and

(3) $U_{j j}=E_{j}$,

for all $1 \leqq j, k, l, m \leqq n$. For each $A$ in $(E \mathscr{A} E) \cdot P_{0}$, there are unique $B_{j k}(1 \leqq j, k \leqq n)$ in $\mathscr{Z}_{1} P_{0}$ such that

$$
A=\sum_{j, k} B_{j k} U_{j k}
$$

We prove that the map $\Psi_{\zeta_{0}}=\Psi$ takes $(E A E) \cdot P_{0}$ onto the set of all linear operators on an $n$-dimensional Hilbert space $H_{n}$. Let $\Psi\left(U_{j k}\right)=V_{j k}(1 \leqq j, k \leqq n)$. If $e_{1}, e_{2}, \ldots, e_{n}$ is an orthonormal basis of $H_{n}$, define $V_{j k} e_{l}=\delta_{j l} e_{k}$. Then for each $A$ in $(E A E) \cdot P_{0}$ we have

$$
\Psi(A)=\sum \alpha_{j k} V_{j k}
$$

where $\alpha_{j k}=\widehat{B}_{j k}\left(\zeta_{0}\right)$ if $A=\sum B_{j k} U_{j k}, B_{j k} \in \mathscr{Z}_{1} P_{0}$; thus $\Psi(A)$ is defined on $H_{n}$. It is easy to see that $\Psi\left((E \mathscr{A} E) \cdot P_{0}\right)$ is the set of all linear functionals on $H_{n}$.

We have for each $A$ in $\mathscr{A}$ that

(1)

$$
\begin{aligned}
f_{\zeta}(A) & =(E A E)^{\# \uparrow\left(\zeta_{0}\right)} \\
& =\left(E A E \cdot P_{0}\right)^{\# \wedge}\left(\zeta_{0}\right) \\
& =\sum_{j}\left(E_{j} A E_{j}\right)^{\# \wedge}\left(\zeta_{0}\right) \\
& =\sum_{j}\left(\Psi(E A E) e_{j}, e_{j}\right) .
\end{aligned}
$$

Now each functional $A \rightarrow\left(E_{j} A E_{j}\right)^{\# \wedge}\left(\zeta_{0}\right)$ is irreducible on $E \mathscr{A} E$. Indeed, $E_{j}$ is abelian in $E A E$. Thus for each $A$ in $\mathscr{A}$ there is a $B$ in $\mathscr{Z}_{1} P_{0}$ such that $E_{j} A E_{j}=B E_{j}$. So

$$
\begin{aligned}
\left(E_{j} A E_{j}\right)^{\#} & =\left(B E_{j}\right)^{\#} \\
& =\left(B U_{k j} U_{j k}\right)^{\#} \\
& =\left(B U_{j k} U_{k i}\right)^{\#}=\left(B E_{k}\right)^{\# .}
\end{aligned}
$$


Thus,

$$
\begin{aligned}
\left(E_{j} A E_{j}\right)^{\# \wedge}\left(\zeta_{0}\right) & =\left[\sum_{k=1}^{n}\left(B E_{k}\right)^{\# \uparrow}\left(\zeta_{0}\right)\right] n^{-1} \\
& =\left[\left(B \sum_{k=1}^{n} E_{k}\right) \# \uparrow\left(\zeta_{0}\right)\right] n^{-1} \\
& =\left[\hat{B}\left(\zeta_{0}\right)\right] n^{-1} \\
& =\left[\tau_{E_{j}}(A)^{\wedge}\left(\zeta_{0}\right)\right] n^{-1}
\end{aligned}
$$

Setting $f_{j \zeta}(A)=\left(E_{j} A E_{j}\right)^{\# \wedge}\left(\zeta_{0}\right)$ we have that $f_{j \zeta}$ is irreducible on $E \mathscr{A} E$. Consequently, $f_{j \zeta}$ is irreducible on $\mathscr{A}$.

We now show that $f_{\zeta}$ has property (5). Let $g$ be the restriction of $f_{\zeta}$ to $E \mathscr{A} E$. It is sufficient to show $g$ is generalized irreducible on $E \mathscr{A} E$. Let $g_{1}$ be a positive functional included in $g$. There is a positive functional $g_{2}$ on $L\left(H_{n}\right)$, the set of all linear operators on $H_{n}$, such that $g_{2} \cdot \Psi=g_{1}$ and $g_{2}$ is included in $\sum\left\{w_{e_{k}} \mid 1 \leqq k \leqq n\right\}$. But $\sum w_{e_{k}}$ is a trace on $L\left(H_{n}\right)$. There is an $A_{0}$ in $(E \mathscr{A} E)^{+}$such that

$$
g_{1}(A)=g_{2}(\Psi(A))=\sum_{k} w_{e_{k}}\left(\Psi\left(A_{0} A\right)\right)=g\left(A A_{0}\right),
$$

for all $A$ in $E \mathscr{A} E$. Thus $g$ is generalized irreducible on $E \mathscr{A} E$. Q.E.D.

The converse of the preceding theorem is contained in the next two theorems.

THEOREM 3.3. Let $\mathscr{A}$ be a von Neumann algebra of Type I with center $\mathscr{Z}$ on a Hilbert space $H$ and let $\nu=\nu_{h}$ be a spectral measure on the spectrum $Z$ of $\mathscr{Z}$. Let $E_{1}, E_{2}, \ldots, E_{n}$ be equivalent orthogonal abelian projections in $\mathscr{A}$ with central support $P$ such that $P h=h$. Let $\tau_{j}(A)=\tau_{E_{j}}(A)(1 \leqq j \leqq n)$ for $A \in \mathscr{A}$ and define $f_{j \zeta}(A)=$ $\lambda_{j} \tau_{j}(A)^{\wedge}(\zeta)(1 \leqq j \leqq n)$ where $\lambda_{j}$ is a strictly positive scalar and $\zeta \in Z$. If we set

$$
f(A)=\sum_{j=1}^{n} \int_{z} f_{j \zeta}(A) d \nu(\zeta)
$$

for all $A$ in $\mathscr{A}$, there are elements $B_{0}$ and $B_{0}^{\prime}$ in $\mathscr{A}^{+}$such that

(1) $B_{0} B_{0}^{\prime}=B_{0}^{\prime} B_{0}=E=E_{1}+E_{2}+\cdots+E_{n}$; and

(2) if $g$ is a positive functional on $\mathscr{A}$ included in $f$, there is an $A_{0}$ in $\mathscr{A}^{+}$such that $g(A)=f\left(B_{0}^{\prime} A_{0} B_{0} A\right)=f\left(A B_{0} A_{0} B_{0}^{\prime}\right)$ for all $A$ in $\mathscr{A}$. If $\lambda_{1}=\lambda_{2}=\cdots=\lambda_{n}=1$, we may take $B_{0}=B_{0}^{\prime}=E$.

Proof. Let $B_{0}=\sum\left\{\lambda_{j}^{1 / 2} E_{j} \mid 1 \leqq j \leqq n\right\}$ and let

$$
B_{0}^{\prime}=\sum\left\{\lambda_{j}^{-1 / 2} \mid E_{j} 1 \leqq j \leqq n\right\} .
$$


Set $f_{1}(A)=f\left(B_{0}^{\prime} A B_{0}^{\prime}\right)=\sum\left\{\left(\tau_{j}(A) h, h\right) \mid 1 \leqq j \leqq n\right\}$ for all $A$ in $\mathscr{A}$. Since $\left(\tau_{j}(A) h, h\right)$ is a normal functional on $E \mathscr{A} E, f_{1}(A)$ is a normal functional on $E \mathscr{A} E$. The support of $f_{1}$ is $E$ and, consequently, $f_{1}$ is a normal functional on $\mathscr{A}$. We show that $f_{1}$ is a trace when restricted to $E \mathscr{A} E$. For each $\zeta$ in the spectrum $Z$ of $\mathscr{Z}$ and for each $A, B$ in $E \mathscr{A} E$ it is sufficient to show that

$$
\sum\left\{\tau_{j}(A B)^{\wedge}(\zeta) \mid 1 \leqq j \leqq n\right\}=\sum\left\{\tau_{j}(B A)^{\wedge}(\zeta) \mid 1 \leqq j \leqq n\right\}
$$

We may assume $\zeta \in\{\zeta \in Z \mid \hat{P}(\zeta)=1\}$. There is an orthonormal basis $e_{1}, e_{2}, \ldots, e_{n}$ for an $n$-dimensional Hilbert space $H_{n}$, such that $\Psi_{\zeta}(E \mathscr{A} E)$ is faithfully representable on $H_{n}$ as the algebra of all linear operators and $\left(\Psi_{\zeta}(A) e_{j}, e_{j}\right)=\tau_{j}(A)^{\wedge}(\zeta)$, for all $A \in E \mathscr{A} E$ and $1 \leqq j \leqq n$. Since $\sum\left\{w_{e} \mid 1 \leqq j \leqq n\right\}$ is a trace on $H_{n}$, we have $\sum\left\{\tau_{j}(B A)^{\wedge}(\zeta) \mid 1 \leqq j \leqq n\right\}=\sum\left\{\tau_{j}(B A)^{\wedge}(\zeta) \mid 1 \leqq j \leqq n\right\}$. Thus, $f_{1}$ is a normal finite trace on $E \mathscr{A} E$.

Let $g$ be a positive functional included in $f$. We have that the functional $g_{1}(A)=$ $g\left(B_{0}^{\prime} A B_{0}^{\prime}\right)$ is included in $f_{1}$. There is an element $A_{0}$ in $(E \mathscr{A} E)^{+}$such that $g_{1}(A)=$ $f_{1}\left(A_{0} A\right)$ for all $A$ in $E \mathscr{A} E$. Thus,

$$
\begin{aligned}
g(A) & =g_{1}\left(B_{0} A B_{0}\right)=f_{1}\left(A_{0} B_{0} A B_{0}\right)=f_{1}\left(B_{0} A B_{0} A_{0}\right) \\
& =f\left(B_{0}^{\prime} A_{0} B_{0} A\right)=f\left(A B_{0} A_{0} B_{0}^{\prime}\right)
\end{aligned}
$$

for all $A$ in $\mathscr{A}$. Q.E.D.

The next theorem is obtained by reducing it to the previous one by repeated use of the following lemma.

Lemma 3.4: Let $\mathscr{Z}$ be a commutative von Neumann algebra and let $Q_{1}, Q_{2}, \ldots$, $Q_{n}$ be projections in $\mathscr{Z}$. There are orthogonal projections $P_{1}, P_{2}, \ldots, P_{m}$ in $\mathscr{Z}$ such that

(1) $\sum\left\{P_{j} \mid 1 \leqq j \leqq m\right\}=\operatorname{lub}\left\{\dot{Q_{j}} \mid 1 \leqq j \leqq n\right\}$;

(2) for each $j(1 \leqq j \leqq n)$ there is a finite subset $\pi_{j}$ of $\{1,2, \ldots, m\}$ such that $k \in \pi_{j}$ implies $P_{k} \leqq Q_{j}$ and $k \notin \pi_{j}$ implies $P_{k} \cdot Q_{j}=0$.

THEOREM 3.5. Let $\mathscr{A}$ be a von Neumann algebra of Type I with center $\mathscr{Z}$ on a Hilbert space $H$. Let $\nu=\nu_{h}$ be a spectral measure on the spectrum $Z$ of $\mathscr{Z}$. For each $\zeta$ in the support $Y$ of $\nu$ and for each $j(1 \leqq j \leqq n)$ let us assume that

(1) $f_{j \zeta}(A)$ is a pure state on $\mathscr{A}$;

(2) $f_{j \zeta}(A)=\hat{A}(\zeta)$, for all $A$ in $\mathscr{Z}$; and

(3) $\zeta \rightarrow f_{j \zeta}(A)$ is continuous on $Y$ for each fixed $A$ in $\mathscr{A}$. Let us set

$$
f(A)=\sum_{j=1}^{n} \int f_{j \zeta}(A) d \nu(\zeta)
$$


for each $A$ in $\mathscr{A}$. Assume $f$ is normal. Consider the hypotheses:

(a) for $1 \leqq j<k \leqq n, \zeta \in Y$

$$
f_{j \zeta}+f_{k \zeta}
$$

is generalized irreducible on $\mathscr{A}$;

(b) hypothesis (a) holds, and for $1 \leqq j<k<l \leqq n, \zeta \in Y$

$$
f_{j \zeta}+f_{k \zeta}+f_{l \zeta}
$$

is generalized irreducible on $\mathscr{A}$.

Then if hypothesis (a) is true, there are elements $B_{0}, B_{0}^{\prime}$ in $\mathscr{A}^{+}$with $B_{0} B_{0}^{\prime}=B_{0}^{\prime} B_{0}=E$, where $E$ is the support of $f$, such that if $g$ is a positive functional on $\mathscr{A}$ included in $f$, there is an element $A_{0}$ in $\mathscr{A}^{+}$such that $g(A)=f\left(B_{0}^{\prime} A_{0} B_{0} A\right)=f\left(A B_{0} A_{0} B_{0}^{\prime}\right)$, for all $A$ in $\mathscr{A}$. If hypothesis (b) holds, $f$ is generalized reducible on $\mathscr{A}$.

Proof. Let

$$
f_{j}(A)=\int f_{j \zeta}(A) d \nu(\zeta) \quad(1 \leqq j \leqq n)
$$

for all $A$ in $\mathscr{A}$. Since $f_{j}(1 \leqq j \leqq n)$ is included in $f, f_{j}$ is a normal functional on $\mathscr{A}$. Further, by Tomita's theorem [4, Theorem $1, \S 40] f_{j}(1 \leqq j \leqq n)$ is centrally irreducible on $\mathscr{A}$. The support $E_{j}$ of $f_{j}(1 \leqq j \leqq n)$ is an abelian projection.

Let $Q_{1}^{\prime}, Q_{2}^{\prime}, \ldots, Q_{n}^{\prime}$ be the central supports of $E_{1}, E_{2}, \ldots, E_{n}$ respectively. Let $P_{1}, P_{2}, \ldots, P_{m}$ be the sequence of projections in $\mathscr{Z}$ which satisfy properties (1) and (2) of the lemma with respect to $Q_{1}^{\prime}, Q_{2}^{\prime}, \ldots, Q_{n}^{\prime}$. For each $j$ consider the projections $P_{j} E_{1}, P_{j} E_{2}, \ldots, P_{j} E_{n}$. There is no loss in generality in assuming $P_{j} E_{1}, P_{j} E_{2}, \ldots, P_{j} E_{r}$ are nonzero and that $P_{j} E_{r+1}=P_{j} E_{r+2}=\cdots=P_{j} E_{n}=0$. Since $P_{j} E_{1}, P_{j} E_{2}, \ldots, P_{j} E_{r}$ are nonzero, we have $P_{j} \leqq Q_{k}^{\prime}$ for $k=1,2, \ldots, r$. Thus, $P_{j} E_{1}, P_{j} E_{2}, \ldots, P_{j} E_{r}$ are abelian projections with central support $P_{j}$. This implies that $P_{j} E_{1}, P_{j} E_{2}, \ldots, P_{j} E_{r}$ are equivalent.

Let us define the functional

$$
\begin{aligned}
f_{p_{j}}(A) & =f\left(P_{j} A\right)=\sum_{k} \int \hat{P}_{j}(\zeta) f_{k \zeta}(A) d \nu(\zeta) \quad(1 \leqq k \leqq r) \\
& =\sum_{k} \int_{z_{j}} f_{k \zeta}(A) d \nu(\zeta) \quad(1 \leqq k \leqq r)
\end{aligned}
$$

where $Z_{j}$ is the open and closed subset of $Z$ given by $Z_{j}=\left\{\zeta \in Z \mid \hat{P}_{j}(\zeta)=1\right\}$. We have that the functionals $f_{1 \zeta}, f_{2 \zeta}, \ldots, f_{r \zeta}\left(\zeta \in Z_{j}\right)$ enjoy property (a) (respectively, property (b)) whenever $f_{1 \zeta}, f_{2 \zeta}, \ldots, f_{r \zeta}(\zeta \in Y$ ) enjoy property (a) (respectively, property (b)). If each $f_{p}$, has the property set forth in the conclusion of the theorem for $\mathscr{A} \cdot P_{j}$ depending on whether the $f_{j \zeta}(1 \leqq j \leqq r)$ satisfy (a) or (b), then it is easy to 
see that $f=\sum\left\{f_{p} \mid 1 \leqq j \leqq m\right\}$ will have the same property for $\mathscr{A}$. So we may assume that the supports $E_{1}, E_{2}, \ldots, E_{n}$ of $f_{1}, f_{2}, \ldots, f_{n}$ are equivalent abelian projections.

Let us assume that $\Psi_{\zeta}(\mathscr{A})(\zeta \in Y)$ is represented as an algebra of continuous linear operators on a Hilbert space $H(\zeta)$ such that $\mathscr{A}(\zeta) \supset C(H(\zeta))$, where $C(H(\zeta))$ is the algebra of completely continuous operators on $H(\zeta)$. Now we have for each $\zeta \in Y$ that $f_{j \zeta}([\zeta])=(0)(1 \leqq j \leqq n)$ by hypothesis (2). Furthermore, for each $\zeta \in Y$ and $1 \leqq j \leqq n$, we have

$$
0=f_{j}\left(I-E_{j}\right)=\int f_{j \zeta}\left(I-E_{j}\right) d \nu(\zeta)
$$

Thus, for $1 \leqq j \leqq n, f_{j \zeta}\left(I-E_{j}\right)=0$ for all $\zeta \in Y-N$, where $N$ is a set of $\nu$-measure zero in $Y$. Now the set $N$ contains no open sets since $v$ is a Radon measure whose support is $Y$. Thus the set $\left\{\zeta \in Y \mid f_{j \zeta}\left(I-E_{j}\right)=0\right\}(1 \leqq j \leqq n)$ is dense in $Y$. Since $\zeta \rightarrow f_{j \zeta}\left(I-E_{j}\right)(1 \leqq j \leqq n)$ is continuous on $Y, f_{j \zeta}\left(E_{j}\right)=f_{j \zeta}(I)(1 \leqq j \leqq n)$ for all $\zeta \in Y$. Since for fixed $\zeta \in Y \Psi_{\zeta}\left(E_{j}\right)(1 \leqq j \leqq n)$ is an abelian projection in $\mathscr{A}(\zeta)$ and since $\mathscr{A}(\zeta)$ is irreducible on $H(\zeta)$, we have that either $E_{j}(\zeta)(1 \leqq j \leqq n)$ is a 1-dimensional projection on $H(\zeta)$ or $E_{j}(\zeta)(1 \leqq j \leqq n)$ is equal to 0 . If $E_{j}(\zeta)=0(1 \leqq j \leqq n), E_{j} \in[\zeta]$ $(1 \leqq j \leqq n)$ and $1=f_{j \zeta}\left(E_{j}\right)=0(1 \leqq j \leqq n)$. Thus $E_{j}(\zeta)(1 \leqq j \leqq n)$ is a 1 -dimensional projection in $H(\zeta)$. We may therefore write $f_{j \zeta}=w_{x_{j}} \cdot \Psi_{\zeta}(1 \leqq j \leqq n)$ where $x_{j}$ $(1 \leqq j \leqq n)$ is a unit vector of $H(\zeta)$.

Consider $f_{j \zeta}+f_{k \zeta}$ for $j \neq k$. Then we have $f_{j \zeta}+f_{k \zeta}=\left(w_{x_{j}}+w_{x_{k}}\right) \cdot \Psi_{\zeta}$. Because $f_{j \zeta}<f_{j \zeta}+f_{k \zeta}$, there is an element $B$ in $\mathscr{A}^{+}$with the property

$$
\begin{aligned}
f_{j \zeta}(A) & =f_{j \zeta}(B A)+f_{k \zeta}(B A) \\
& =f_{j \zeta}(A B)+f_{k \zeta}(A B)
\end{aligned}
$$

for all $A$ in $\mathscr{A}$ (hypothesis (a)). Then

$$
\begin{aligned}
w_{x_{j}}[A(\zeta)] & =w_{x_{j}}[B(\zeta) A(\zeta)]+w_{x_{k}}[B(\zeta) A(\zeta)] \\
& =w_{x_{j}}[A(\zeta) B(\zeta)]+w_{x_{k}}[A(\zeta) B(\zeta)]
\end{aligned}
$$

for all $A$ in $\mathscr{A}$. Since $\mathscr{A}(\zeta) \supset C[H(\zeta)]$, we have that

(1) $B(\zeta)^{1 / 2} x_{j}=\lambda_{j} x_{j}$ and $B(\zeta)^{1 / 2} x_{k}=\lambda_{k} x_{j}$, where $\lambda_{j}$ and $\lambda_{k}$ are scalars. Indeed the set $\mathscr{B}=\left\{A_{1}(\zeta) \in \mathscr{A}(\zeta) \mid\left(w_{x_{j}}+w_{x_{k}}\right)\left[A_{1}(\zeta) A(\zeta)\right]=\left(w_{x_{j}}+w_{x_{k}}\right)\left[A(\zeta) A_{1}(\zeta)\right]\right.$, for all $A(\zeta)$ in $\mathscr{A}(\zeta)\}$ is a uniformly closed $*$-subalgebra of $\mathscr{A}(\zeta)$ with identity. Thus $B(\zeta) \in \mathscr{B}$ and $B(\zeta) \geqq 0$ implies $B(\zeta)^{1 / 2} \in \mathscr{B}$. This means $\left(A(\zeta) x_{j}, x_{j}\right)=\left(A(\zeta) B(\zeta)^{1 / 2} x_{j}, B(\zeta)^{1 / 2} x_{j}\right)$ $+\left(A(\zeta) B(\zeta)^{1 / 2} x_{k}, B(\zeta)^{1 / 2} x_{k}\right)$, for all $A(\zeta) \in \mathscr{A}(\zeta)$.

From this expression (1) follows. Thus,

$$
B(\zeta) x_{j}=\lambda_{j}^{2} x_{j} \quad \text { and } \quad B(\zeta) x_{j}=\lambda_{j} \lambda_{k} x_{j} .
$$


We have

$$
\left(A(\zeta) x_{j}, x_{j}\right)=\lambda_{j}^{2}\left(A(\zeta) x_{j}, x_{j}\right)+\lambda_{j} \lambda_{k}\left(A(\zeta) x_{j}, x_{k}\right)
$$

for all $A$ in $\mathscr{A}$. So

$$
x_{j}=\bar{\lambda}_{j}^{2} x_{j}+\bar{\lambda}_{j} \bar{\lambda}_{k} x_{k} .
$$

Because $B(\zeta)^{1 / 2} \geqq 0, \lambda_{j}\left(x_{j}, x_{j}\right)=\left(B(\zeta)^{1 / 2} x_{j}, x_{j}\right) \geqq 0$ and so $\lambda_{j} \geqq 0$. We also have that $\lambda_{j}>0$ since $\left(x_{j}, x_{j}\right)=f_{j \zeta}\left(E_{j}\right) \neq 0$ (expression (2)). Therefore (from expression (2)),

$$
\left(1-\lambda_{j}^{2}\right) x_{j}=\lambda_{j} \lambda_{k} x_{k} \text {. }
$$

Either $x_{j}$ is a scalar multiple of $x_{k}$ or $\lambda_{k}=0$. If $\lambda_{k}=0$,

$$
\begin{aligned}
\lambda_{j}\left(x_{j}, x_{k}\right) & =\left(B(\zeta)^{1 / 2} x_{j}, x_{k}\right) \\
& =\left(x_{j}, B(\zeta)^{1 / 2} x_{k}\right) \\
& =\bar{\lambda}_{k}\left(x_{j}, x_{j}\right) \\
& =0
\end{aligned}
$$

by expression (1). This means that $x_{j}$ and $x_{k}$ are orthogonal since $\lambda_{j}>0$. Since $E_{j}(\zeta)$ and $E_{k}(\zeta)$ are both 1-dimensional projections with $E_{j}(\zeta) x_{j}=x_{j} \neq 0$ and $E_{k}(\zeta) x_{k}=x_{k} \neq 0$, we have $E_{j}(\zeta)$ is orthogonal to $E_{k}(\zeta)$ if $\lambda_{k}=0$ and $E_{j}(\zeta)=E_{k}(\zeta)$ if $\lambda_{k} \neq 0$.

Now by the preliminary remarks the map $\zeta \rightarrow\left\|\left(E_{j} \cdot E_{k}\right)(\zeta)\right\|=\left\|E_{j}(\zeta) E_{k}(\zeta)\right\|$ is continuous on $Y$. Since $E_{j}(\zeta)=E_{k}(\zeta)$ or $E_{j}(\zeta) \cdot E_{k}(\zeta)=0$ for each $\zeta \in Y$, the function $\left\|\left(E_{j} \cdot E_{k}\right)(\zeta)\right\|$ assumes at most two values, namely 0 and 1 . Hence the set

$$
X_{j k}=\left\{\zeta \in Y \mid E_{j}(\zeta)=E_{k}(\zeta)\right\}
$$

is open and closed in $Y$ and hence in $Z$ since $Y$ is open and closed in $Z$. Let $P$ be the projection in $\mathscr{Z}$ with the property

$$
\left\{\zeta \in Z \mid P^{\wedge}(\zeta)=1\right\}=Y
$$

and let $Q_{j k}$ be the projection in $\mathscr{Z} P$ with the property

$$
X_{j k}=\left\{\zeta \in Z \mid Q_{j k}(\zeta)=1\right\}
$$

Such a projection $Q_{j k}$ exists because $X_{j k} \subset Y$.

Suppose we have found projections $Q_{j k}$ with the properties just specified for all $1 \leqq j<k \leqq n$. Consider the set of projections,

$$
S=\left\{Q_{j k} \mid 1 \leqq j<k \leqq n\right\} \cup\left\{P-Q_{j k} \mid 1 \leqq j<k \leqq n\right\}
$$

in $\mathscr{Z} P$. There are projections $Q_{1}, Q_{2}, \ldots, Q_{r}$ in $\mathscr{Z} P$ which satisfy (1) and (2) of the lemma with respect to the set $S$. 
For arbitrary $Q_{l}$ in $\left\{Q_{1}, Q_{2}, \ldots, Q_{r}\right\}$ consider the set $Q_{l} E_{1}, Q_{l} E_{2}, \ldots, Q_{l} E_{n}$. Since $E_{1} \sim E_{2} \sim \cdots \sim E_{n}$, we have $Q_{l} E_{1} \sim Q_{l} E_{2} \sim \cdots \sim Q_{l} E_{n}$. For each $j \neq k, Q_{l} E_{j}$ is orthogonal to $Q_{l} E_{k}$ or is equal to $Q_{l} E_{k}$. Indeed, either $Q_{l} \leqq Q_{j k}$ or $Q_{l} \leqq P-Q_{j k}$. In the former case $\left\|\left(E_{j}-E_{k}\right)(\zeta)\right\|=0$ for all $\zeta$ in $Z_{l}=\left\{\zeta \in Y \mid Q_{\imath}(\zeta)=1\right\}$. Thus, $\left\|\left(\left(E_{j}-E_{k}\right) \cdot Q_{l}\right)(\zeta)\right\|=0$ for all $\zeta$ in $Z$. Since

$$
\bigcap\{[\zeta] \mid \zeta \in Z\}=0
$$

we have $Q_{l} E_{j}=Q_{l} E_{k}$. In the latter case, on the other hand,

$$
\left\|\left(Q_{l} \cdot\left(E_{j} \cdot E_{k}\right)\right)(\zeta)\right\|=0
$$

for all $\zeta \in Z$, and therefore $Q_{l} E_{j}$ and $Q_{l} E_{k}$ are orthogonal.

We have

$$
f_{Q_{l}}(A)=f\left(Q_{l} A\right)=\sum_{j=1}^{n} \int_{z_{l}} f_{j \zeta}(A) d v(\zeta),
$$

for all $A$ in $\mathscr{A}$. We divide the set $\{1,2, \ldots, n\}$ into disjoint parts $\pi_{1}, \pi_{2}, \ldots, \pi_{m}$ such that (1) $\bigcup_{k} \pi_{k}=\{1,2, \ldots, n\} ;$ (2) $j$ and $j^{\prime}$ in $\pi_{k}$ implies $Q_{l} E_{j}=Q_{l} E_{j^{\prime}}$; and (3) $j \in \pi_{k}$ and $j^{\prime} \in \pi_{k^{\prime}}\left(k \neq k^{\prime}\right)$ implies $Q_{l} E_{j}$ is orthogonal to $Q_{l} E_{j^{\prime}}$. We have

$$
f_{Q_{\ell}}(A)=\sum_{j=1}^{m} \int \lambda_{j} g_{j \zeta}(A) d \nu(\zeta),
$$

for all $A$ in $\mathscr{A}$. Here, $g_{j 5}(A)=f_{k \zeta}(A)$ where $k \in \pi_{j}(1 \leqq j \leqq m)$ and $\lambda_{j}$ is a scalar equal to the cardinality of $\pi_{j}(1 \leqq j \leqq m)$. Since $f_{k \zeta}(A)=\tau_{Q_{l} E_{k}}(A)^{\wedge}(\zeta)=\tau_{Q_{l} E_{k^{\prime}}}(A)^{\wedge}(\zeta)=$ $f_{k^{\prime} \xi}(A)$ for $k, k^{\prime} \in \pi_{j}$ (because $Q_{l} E_{k}=Q_{l} E_{k^{\prime}}$ ), the functionals $g_{j \zeta}$ are uniquely defined.

If each $f_{Q_{l}}(l=1,2, \ldots, r)$ possesses the properties set forth in either conclusion for (a) and (b) with regard to $\mathscr{A} Q_{l}$, then $f=\sum_{l} f_{Q_{l}}$ possesses the same properties with regard to $\mathscr{A}$. Thus, we may assume that

$$
f(A)=\sum_{j=1}^{n} \int \lambda_{j} \tau_{E_{j}}(A)^{\wedge}(\zeta) d \nu(\zeta),
$$

for all $A$ in $\mathscr{A}$, where $E_{1}, E_{2}, \ldots, E_{n}$ are orthogonal equivalent abelian projections. Thus, we have reduced to Theorem 3.3.

Let $E=E_{1}+E_{2}+\cdots+E_{n}$; let $B_{0}$ be the element in $\mathscr{A}^{+}$given by

$$
B_{0}=\lambda_{1}^{1 / 2} E_{1}+\lambda_{2}^{1 / 2} E_{2}+\cdots+\lambda_{n}^{1 / 2} E_{n}
$$

and let

$$
B_{0}^{\prime}=\lambda_{1}^{-1 / 2} E_{1}+\lambda_{2}^{-1 / 2} E_{2}+\cdots+\lambda_{n}^{-1 / 2} E_{n} .
$$


If $g$ is a positive functional on $\mathscr{A}$ included in $f$, by Theorem 3.3 there is an $A_{0}$ in $\mathscr{A}^{+}$such that

$$
g(A)=f\left(B_{0}^{\prime} A_{0} B_{0} A\right)=f\left(A B_{0} A_{0} B_{0}^{\prime}\right),
$$

for all $A$ in $\mathscr{A}$. This completes the proof of part (a).

Let us assume that (b) applies. Using hypothesis (a), we can reduce to the form (3)

$$
f_{Q_{l}}(A)=\sum_{j=1}^{m} \int \lambda_{j} g_{j \zeta}(A) d \nu(\zeta)
$$

We shall show either $m=1$ or $\lambda_{1}=\lambda_{2}=\cdots=\lambda_{m}=1$. In both of these situations Theorem 3.3 can be applied to give the desired conclusion.

We argue by contradiction. Assume $\lambda_{1}>1$ and $m \neq 1$. In the notation of the proof

$$
2 f_{1 \zeta}(A)+f_{2 \zeta}(A)=\left(2 w_{x_{1}}+w_{x_{2}}\right)[A(\zeta)]
$$

is generalized irreducible. Here $x_{1}$ and $x_{2}$ are orthogonal unit vectors in $H(\zeta)$. This is impossible as the next proposition will demonstrate.

Proposition 3.6. Let $\mathscr{A}$ be a $B^{*}$-algebra with identity operating on a Hilbert space $H$. Assume $\mathscr{A} \supset C(H)$, where $C(H)$ is the algebra of completely continuous operators on $H$. If $x_{1}$ and $x_{2}$ are orthogonal unit vectors in $H$, then there is a positive functional $g$ on $\mathscr{A}$ included in $2 w_{x_{1}}(A B)+w_{x_{2}}(A B)$, for all $A$ in $\mathscr{A}$, implies $B \neq B^{*}$.

Proof. Let $g=w_{y}$ where $y=x_{1}+x_{2}$. If $A \in L(H)^{+}$, we have

$$
\left(A^{1 / 2}\left(x_{1}-x_{2}\right), A^{1 / 2}\left(x_{1}-x_{2}\right)\right) \geqq 0,
$$

and so

$$
\left(A^{1 / 2} x_{1}, A^{1 / 2} x_{1}\right)+\left(A^{1 / 2} x_{2}, A^{1 / 2} x_{2}\right) \geqq\left(A^{1 / 2} x_{1}, A^{1 / 2} x_{2}\right)+\left(A^{1 / 2} x_{2}, A^{1 / 2} x_{1}\right) .
$$

Therefore, we have

$$
(A y, y) \leqq 2\left(A^{1 / 2} x_{1}, A^{1 / 2} x_{1}\right)+2\left(A^{1 / 2} x_{2}, A^{1 / 2} x_{2}\right) \leqq 2\left(2 w_{x_{1}}(A)+w_{x_{2}}(A)\right) .
$$

Thus, $w_{y}<2 w_{x_{1}}+w_{x_{2}}$ on $L(H)$ and thus on $\mathscr{A}$. Let $B \in L(H)$ have the property

$$
w_{y}(A)=2 w_{x_{1}}(A B)+w_{x_{2}}(A B)
$$

for all $A$ in $\mathscr{A}$. Let $A_{1} x_{1}=0, A_{1} x_{2}=x_{1}$ and $A_{1}(K)=(0)$ where $K$ is the orthogonal complement of the subspace of $H$ generated by $x_{1}$ and $x_{2}$; let $A_{2} x_{1}=x_{2}, A_{2} x_{2}=0$ and $A_{2}(K)=(0)$. We have $A_{1}, A_{2} \in C(H) \subset A$. Then

$$
\begin{aligned}
1=w_{y}\left(A_{1}^{*}\right) & =2 w_{x_{1}}\left(A_{1}^{*} B\right)+w_{x_{2}}\left(A_{1}^{*} B\right) \\
& =0+\left(B x_{2}, x_{1}\right)
\end{aligned}
$$


and

$$
\begin{aligned}
1=w_{y}\left(A_{2}^{*}\right) & =2 w_{x_{1}}\left(A_{2}^{*} B\right)+w_{x_{2}}\left(A_{2}^{*} B\right) \\
& =2\left(B x_{1}, x_{2}\right)+0 .
\end{aligned}
$$

So

$$
\left(B x_{1}, x_{2}\right)=\frac{1}{2} \quad \text { and } \quad\left(B x_{2}, x_{1}\right)=1
$$

We have

$$
\left(x_{1}, B x_{2}\right)=\overline{\left(B x_{2}, x_{1}\right)}=1 \text {. }
$$

Thus $B \neq B^{*}$. Q.E.D.

\section{BIBLIOGRAPHY}

1. J. Dixmier, Les algebres d'operateurs dans l'espace hilbertien, Gauthier-Villars, Paris, 1957.

2. J. Glimm, A Stone-Weierstrass Theorem for $C^{*}$-algebras, Ann. of Math. 72 (1960), 216-244.

3. —, Type I $C^{*}$-algebras, Ann. of Math. 74 (1961), 572-612.

4. M. A. Naimark, Normed rings, GITTL, Moscow, 1956. (Russian)

5. C. Rickart, General theory of Banach algebras, Van Nostrand, Princeton, N. J., 1960.

6. J. Taylor, Tomita decomposition of rings of operators, Manuscript.

ILLINOIS INSTITUTE OF TeChNOLOGY,

Chicago, Illinois 